\title{
Influence of cell interactions in a novel model of postnatal mucosal regeneration
}

\author{
H R H Patel, I S Tait, G S Evans, F C Campbell
}

\begin{abstract}
Background and aims-Conventional models of postnatal mucosal regeneration are cumbersome and limited: a novel model is described here. In addition, the influence of cell interactions on mucosal regeneration is examined within the model.

Methods-Postnatal rat small intestinal mucosa was digested by enzymes to yield heterotypic cell aggregates (CA). CA colony forming ability, growth, and limited cytodifferentiation were assessed in vitro. CA were transplanted subcutaneously and retrieved for histological examination at staggered intervals to assess neomucosal morphogenesis and cytodifferentiation in vivo. Cell interactions in CA were disrupted by enzymes, thus producing cell suspensions (CS). Regeneration by CA and CS were compared.
\end{abstract}

Results-CA produced proliferative colonies in vitro and showed a temporal sequence of neomucosal morphogenesis and differentiation in vivo. CA colonies were more numerous within 24 hours of primary culture and had greater cellularity by 96 hours than CS colonies. Alkaline phosphatase was expressed only by 258 of 696 CA colonies (37\%). CA subcutaneous grafts (48 of $56(87 \%)$ ) regenerated small intestinal neomucosa while CS were unsuccessful.

Conclusion-These methods provide a model of mucosal regeneration which includes constituent processes of colony formation, growth, neomucosal morphogenesis, and cytodifferentiation. Preservation of cell interactions within $C A$ seems advantageous to regeneration within the model.

University Department of Surgery, Ninewells Hospital and Medical School, Dundee H R H Patel I S Tait

F C Campbell

CRC Department of Epithelial Biology, Paterson Institute for Cancer Research Christie Hospital, Manchester G S Evans

Correspondence to: Mr F C Campbell, University Department of Surgery, Ninewells Hospital Surgery, Ninewells Hospital and Medical School, Dundee

Accepted for publication 20 November 1995 Typically, surviving crypt epithelium within the injured area proliferates to form undifferentiated epithelial colonies. $^{1-4}$ Colonies enlarge then coalesce to restore epithelial cover. ${ }^{56}$ Epithelium also interacts with

The continuity and absorptive function of the tion. This phenomenon is a coordinated mesenchyme in the remodelling process of neomucosal morphogenesis to restore a three dimensional crypt/villus architecture. Epithelial cytodifferentiation provides distinct cell lineages, expressing specific enzymes and transporter proteins. ${ }^{7}$ By these processes regeneration induces a new mucosa which is indistinguishable from parent tissue. ${ }^{5}$

Conventional models of mucosal regeneration involve applied injury to whole intestine in vivo, by external irradiation or systemic administration of cytotoxic drugs. ${ }^{1-4}$ These methods induce mucosal damage and subsequent regeneration in vivo but are cumbersome and limited by toxicity. This study seeks to investigate methods of primary culture and grafting of disaggregated postnatal mucosa which we have previously described, ${ }^{78}$ as a model of mucosal regeneration.

Factors which influence mucosal regeneration are obscure. This study has also investigated the influence of cell interactions upon the constituent processes of regeneration within the model system.

\section{Methods}

EPITHELIAL ISOLATION

Postnatal small intestinal mucosa from AO/OLA inbred rats (aged 6-8 days) was digested by enzymes to yield cell aggregates (CA). Cell aggregates were subsequently digested to cell suspensions (CS) in order to assess the influence of cell interactions on regeneration.

\section{$\mathrm{CA}$}

CA were obtained as previously described by Evans et al. ${ }^{9}$ Briefly, small intestinal segments were diced, washed thoroughly in Hanks buffered salt solution (HBSS - Gibco Life Technologies Ltd, Paisley, Scotland), then incubated in a combination of type I dispase $0.1 \mathrm{mg} / \mathrm{ml}$ (Boehringer Mannheim - Lewes, England) and crude collagenase $300 \mathrm{U} / \mathrm{ml}$ (type XI - Sigma, Poole, England) for 20 minutes at room temperature. The preparation underwent differential sedimentation in Dulbecco's modified Eagle's medium (DMEM - Gibco BRL, Poole, England) with $2 \%$ sorbitol and $5 \%$ fetal calf serum (FCS Gibco BRL). This procedure yields CA of proliferative crypt epithelium in morphological contact with adjacent mesenchymal elements. ${ }^{89}$ These aggregates of heterotypic cells were prepared from four to six neonatal rat intestines during a typical isolation, then resuspended in $20 \mathrm{ml} \mathrm{DMEM}$ + sorbitol +FCS 
at $4^{\circ} \mathrm{C}$ and gently agitated. The number of $\mathrm{CA}$ were counted $5 \times$ aliquots of $100 \mu l$ under phase contrast microscopy to give an estimate of the total number within the isolation. Further serial dilutions were performed as necessary.

CS

CS were prepared by submitting CA from the above procedure to an additional digestion step. CA were incubated in a combination of $1 \%$ trypsin (Sigma) and $2 \%$ ethylene-diaminetetra acetic acid (EDTA); (Sigma) for 30 minutes at room temperature. Preparations of CS were examined by phase contrast microscopy to confirm complete disaggregation.

Assessment of cell number within $C A$

The number of $\mathrm{CA}$ were counted in $5 \times$ aliquots of $100 \mu l$ under phase contrast microscopy. After digestion of CA to single cells by trypsin and EDTA as outlined above, the number of disaggregated cells was counted under phase contrast microscopy using a haemocytometer. This gave an estimate of the number of cells per cell aggregate.

PRIMARY CULTURE OF DISAGGREGATED

POSTNATAL INTESTINAL EPITHELIUM

Disaggregated postnatal intestinal epithelium was cultured in 24 multiwell plates, which had been precoated with a layer of $10 \%$ bovine dermal collagen (Vitrogen, Imperial Laboratories, Andover, England). These primary cultures were maintained in high glucose formulation DMEM (Gibco BRL) containing $10 \%$ FCS, sodium pyruvate (Sigma), glutamine (Gibco), insulin (Sigma), epidermal growth factor (EGF; Sigma), transferrin, streptomycin ( $60 \mu \mathrm{gm} / \mathrm{ml}$; Evans Medical Ltd), and penicillin (100 U/ml; Crystapen, Glaxo). All cultures were incubated at $37^{\circ} \mathrm{C}$ in a humidified atmosphere in a $7 \cdot 5 \% \quad \mathrm{CO}_{2}$ incubator (Herareus - Brentwood, Essex).

\section{ASSESSMENT OF COLONY FORMING ABILITY} AND COLONY CELLULARITY IN VITRO

CA were suspended in serial dilutions of culture medium to a final concentration of $30 \mathrm{CA} / \mathrm{ml}$. Equivalent suspensions of CS were prepared. Suspensions were agitated to ensure even dispersion and $1 \mathrm{ml}$ of each was added to each well of 24 multiwell plates. This gave an average of $30 \mathrm{CA}$ per well or the equivalent of CS. After plating the preparations in primary culture, the number of colonies per well was assessed by phase contrast microscopy at 24 hours. In this study, an epithelial colony was defined as a localised cluster of 10 or more chromophilic cells which had attached in primary culture. To estimate proliferation, colony cellularity was assessed at both 24 and 96 hours. Cellularity was assessed by counting cells in all colonies, using a microscope incorporating an eyepiece graticule.

\section{ASSESSMENT OF CYTODIFFERENTIATION IN}

VITRO

Epithelial differentiation was sought in vitro by histochemistry for alkaline phosphatase, which is expressed by absorptive enterocytes, and for mucin expressed by goblet cells. Cultures were stained using the fluorescent substrate Vector red in $100 \mathrm{mM}$ Tris- $\mathrm{HCl}$ buffer at $\mathrm{pH} 8.2$ (Vector Laboratories Ltd, Peterborough, UK), for expression of alkaline phosphatase and viewed under a fluorescein filter. The number of colonies with a positive stain was expressed as a percentage of the total. Goblet cells were sought by staining of cultures with periodic acid Schiff (PAS) and examination under light microscopy.

\section{ASSESSMENT OF CELL VIABILITY}

Viability of cells within the isolates or primary cultures was estimated using the MTT (3-(4,5-)dimethylthiazol-2-yl)-2,5-ditetrazolium bromide) assay. The assay relies on the conversion of MTT to a dark blue coloured precipitate by mitochondrial succinate dehydrogenase and estimates the viability of a known number of cells. ${ }^{10}$ Immediately after isolation, 30 cell aggregates or the equivalent number of isolated cells within cell suspensions were plated into 24 well multiwell plates and incubated for one hour at $37^{\circ} \mathrm{C}$ with MTT $(5 \mathrm{mg} / \mathrm{ml})$ in phosphate buffered saline (PBS). The blue precipitate formed from MTT was dissolved in $0.04 \mathrm{M}$ acid-isopropanol and measured at $570 \mathrm{~nm}$ (wavelength) absorbance by a spectrophotometer (Pye Unicam SP8-100 UV/VIS). This assessment was also carried out after 24 and 48 hours of primary culture.

\section{IN VIVO TRANSPLANTATION OF}

DISAGGREGATED EPITHELIUM

Disaggregated epithelium was centrifuged at low speed (300-400 revolutions per minute) to produce pellets for subcutaneous grafting. In recipient adult AO/OLA rats, small dorsal skin flaps were raised and subcutaneous tunnels were created by blunt dissection. Aliquots of $20 \mu \mathrm{l} /$ cell pellet/graft were then seeded into each tunnel using a sterile micropipette within two hours of the isolation procedure. ${ }^{78}$ Sham operations involved dorsal skin flaps and creation of subcutaneous tunnels, without any cellular graft. These procedures were identical for both CA and CS grafts.

\section{Assessment of neomucosal morphogenesis}

Forty CA grafts were carried out for the study of the temporal sequence of neomucosal morphogenesis. Successful grafts could be identified in live recipients at early stages by a palpable subcutaneous lump (unpublished observation). Palpable grafts $(n=8)$ were retrieved at each staggered interval of 3, 5, 7,10 , and 14 days after transplantation. Temporal changes in graft morphology and cytology were assessed by histological examination (see below). 
Comparison of graft success by $C A$ versus $C S$ Sixteen CA grafts, 16 CS grafts, and 20 control sham operations were carried out in this assessment. All grafts were harvested at 14 days irrespective of whether a lump could be palpated. Where there was no lump, the skin and subcutaneous tissue of the graft site was harvested and examined histologically.

A total of 56 CA grafts, 16 CS grafts, and 20 control sham operations were carried out.

\section{SMALL ASSESSMENT}

All specimens were submitted for histological, histochemical, and immunohistochemical assessment after fixation in methacarn $(10 \%$ acetic acid, $30 \%$ chloroform and $60 \%$ methanol), dehydrated, and embedded in paraffin wax. Sections were cut serially at 5 $\mu \mathrm{m}$, mounted on glass microscope slides, and assessed. Haematoxylin and eosin staining was used for assessment of graft morphology.

\section{Cell lineage studies}

Each of the four principal small intestinal epithelial cell lineages was sought in retrieved grafts.

Absorptive enterocytes. Small intestinal absorptive enterocytes were identified by (a) histochemistry for alkaline phosphatase activity using the Vector red substrate (Vector Laboratories) and microscopy with a fluorescein filter as described ${ }^{7}$ and (b) immunohistochemistry for the digestive enzyme sucrase. For sucrase expression, grafts were retrieved and flash frozen in liquid nitrogen. All solutions were prepared in PBS. Frozen sections $(5 \mu \mathrm{M})$ were fixed in $4 \%$ paraformaldehyde for 10 minutes, washed in PBS, then stained with mouse monoclonal antibodies to rat sucrase (generous gift from Dr A Quaroni, Cornell University, Ithaca, NY, USA). The primary antibodies were applied at a $1 / 50$ dilution containing $0.5 \%$ bovine serum albumin (BSA) and 5\% non-immune goat serum, for two hours at $20^{\circ} \mathrm{C}$. Sections were washed three times in PBS, and a secondary goat anti-mouse IgG Texas red (Vector Laboratories) was applied at $1 / 100$ dilution in PBS $+0.5 \%$ BSA $+5 \%$ non-immune rat serum, for one hour at $20^{\circ} \mathrm{C}$. The sections were then washed again three times in PBS and counterstained with $2.5 \mu \mathrm{g} / \mathrm{ml}$ Hoescht 33258 (Sigma) dye for five minutes, washed finally in distilled water, and mounted in Glycergel $0.25 \mu \mathrm{g} / \mathrm{ml}$ (Dako - High Wycombe, England). Staining was observed using a rhodamine filter.

Goblet cells. Goblet cells were sought by mucin histochemistry using the PAS stain.

Entero-endocrine cells. These cells were identified by immunohistochemical staining using a rabbit polyclonal anti-serotonin antibody (Serotec, Oxford, UK). Sections were dewaxed, endogenous peroxidase activity was blocked in methanol with $0.3 \% \mathrm{H}_{2} \mathrm{O}_{2}$ and $0.01 \mathrm{M} \mathrm{HCl}$ for 20 minutes, and they were then washed in PBS. The primary antibody was applied at a 1600 dilution in PBS with $0.5 \%$ bovine serum albumin and $5 \%$ non-immune goat serum overnight at $4^{\circ} \mathrm{C}$. The sections were then washed three times in PBS, and a biotinylated goat anti-rabbit polyclonal antibody (Vector Laboratories) was applied at $1 / 200$ dilution in PBS with $0.5 \%$ BSA for one hour at $20^{\circ} \mathrm{C}$. The sections were then washed three times in PBS and a 1/100 dilution of avidin-biotin peroxidase (Vector Laboratories) was applied in PBS with $0.5 \%$ BSA for one hour at $20^{\circ} \mathrm{C}$. The sections were washed again three times in PBS and the antibody binding sites were revealed by incubating the sections for five minutes in a solution of $0.2 \mathrm{mg} / \mathrm{ml}$ diaminobenzidine hydrochloride (DAB), $0 \cdot 1$ $\mathrm{mg} / \mathrm{ml} \mathrm{NiCl}$ and $0.03 \% \mathrm{H}_{2} \mathrm{O}_{2}$ in PBS.

Paneth cells. Paneth cells were identified by the above immunohistochemical procedure using a rabbit polyclonal anti-lysozyme antibody (Dako, High Wycombe, England), at a dilution of $1 / 250$.

\section{STATISTICAL ANALYSIS}

Summary statistics were expressed as the median (SD). Hypotheses were tested using the Mann-Whitney $U$ test for unpaired data. In the CA and CS groups, colony formations were compared at 24 hours while colony cellularity was evaluated at 24 and 96 hours. MTT absorbance values of CA and CS were compared at 0,24 , and 48 hours after disaggregation.

\section{Results}

EPITHELIAL YIELD

Each pellet measured between $160-220 \mu l$ and contained $120-172 \times 10^{3}$ cell aggregates $(\mathrm{CA}$; Fig 1A), corresponding to $30-40 \times 10^{3} \mathrm{CA}$ per
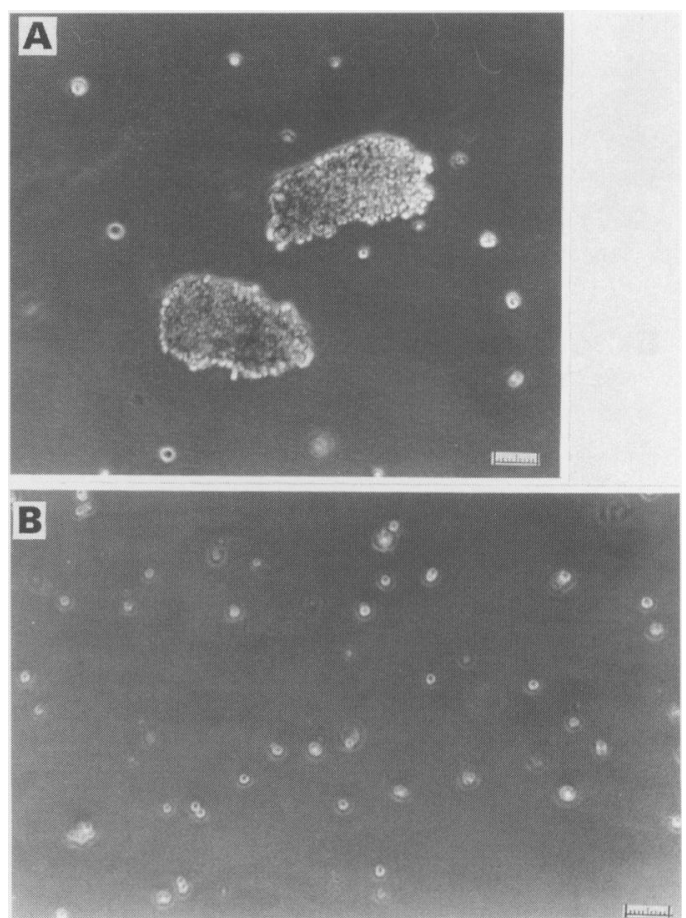

Figure 1: Cell preparations after disaggregation of neonatal intestine. (A) Cell aggregates are shown after collagenase/dispase disaggregation (bar, $120 \mu \mathrm{m}$ ). (B) These were further digested by incubation in a solution of trypsin/EDTA to yield single cell suspensions (bar, 120 $\mu \mathrm{m}$, magnification $\times 120$ ). 


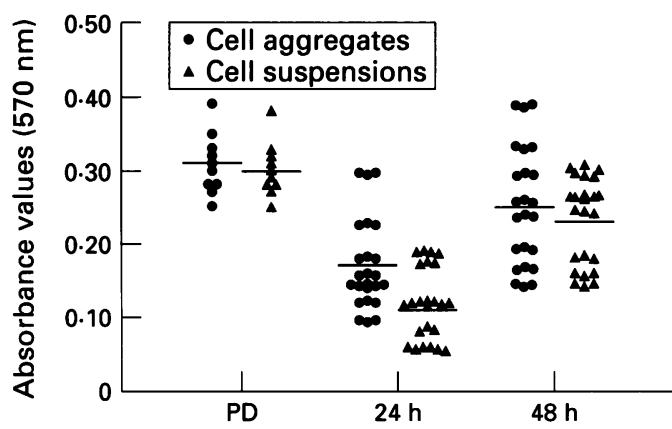

Growth time of primary cultures

Figure 2: Cell viability in colonies raised from cell aggregates and single cell suspensions assessed by MTT (3-(4,5-) dimethylthiazole-2-yl)-2,5-di-tetrazolium bromide) assay. Relative absorbance values at $570 \mathrm{~nm}$ (wavelength) are shown immediately post disaggregation (PD) and at 24 and 48 hours thereafter. Median values are denoted by horizontal lines.

retrieved intestine. Further disaggregation of CA by trypsin and EDTA yielded preparations of predominantly single cells in suspension (CS; Fig 1B). Cell aggregates contained median (SD) 800 (130) cells each. Values are approximate.
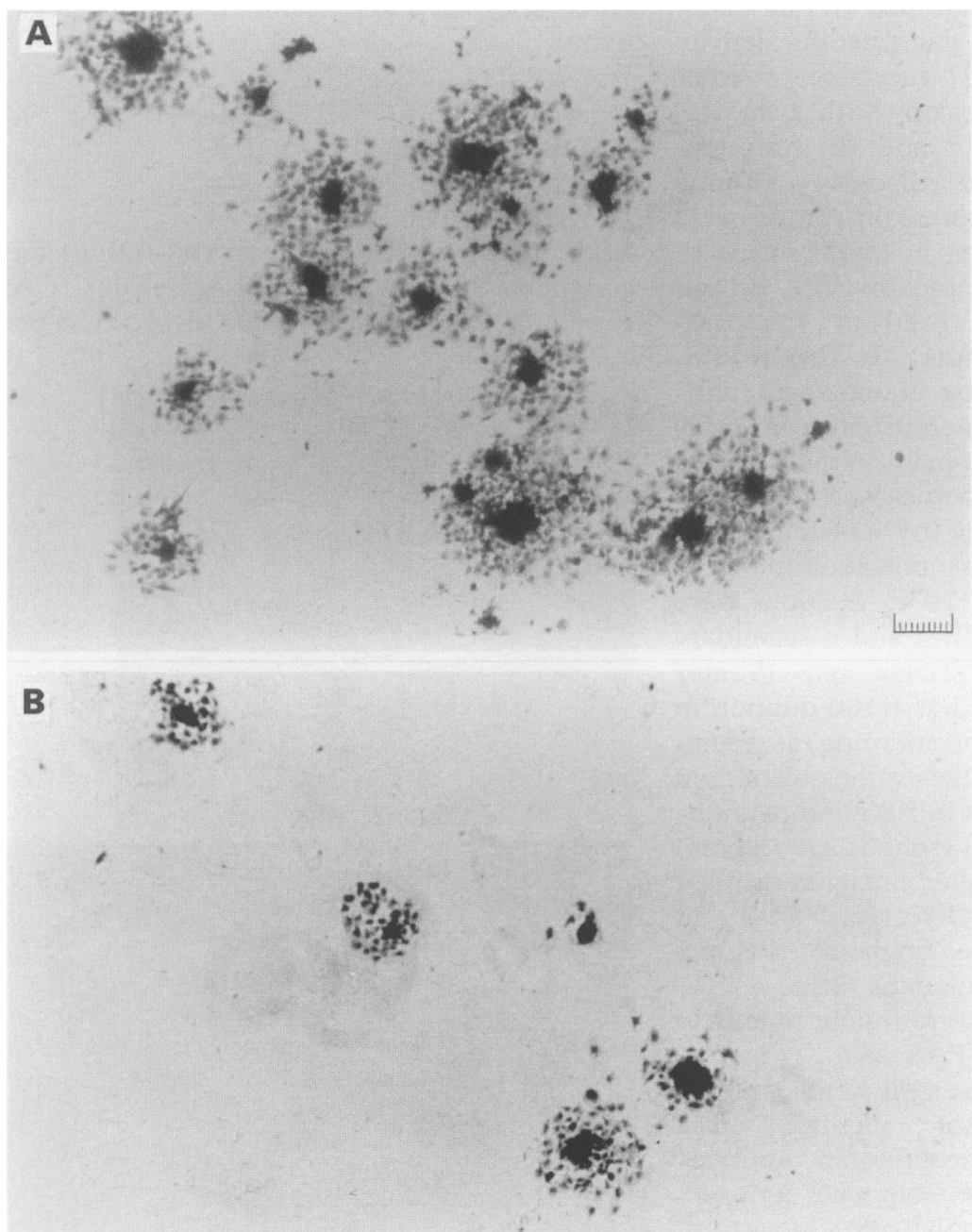

Figure 3: Colonies raised from $(A)$ cell aggregates or $(B)$ single cell suspensions at 24 hours after plating. Note the greater number and cellularity of colonies from cell aggregates (magnification $\times 75 ;$ bar, $1200 \mu \mathrm{m}$ ).
Colony formation and cellularity of cell aggregates (CA) versus cell suspensions (CS) at 24 and 96 hours after plating in primary culture

\begin{tabular}{lrr}
\hline & $C A$ & $C S$ \\
\hline Colonies per well: & $29(10)$ & $8(5)^{\star}$ \\
24 h & & \multicolumn{1}{l}{ hells per colony: } \\
$24 \mathrm{~h}$ & $23(10)$ & $14(6)^{\star}$ \\
$96 \mathrm{~h}$ & $178(48)$ & $123(50)^{\star}$ \\
\hline
\end{tabular}

Values - median (SD). ${ }^{\star} \mathrm{p}<0.05$, Mann-Whitney unpaired U test.

\section{IN VITRO STUDIES}

Cell viability as assessed by the MTT assay, was similar in both CA and CS immediately after digestion (Fig 2). Absorbance values declined in both groups after 24 hours but subsequently increased with cell attachment and growth (Fig 2). CA formed 29 (10) colonies per well compared with 8 (5) for CS p $<0.001$, after 24 hours of primary culture. At 24 hours primary culture, CA had formed colonies with greater cellularity than CS (Fig 3A, B; Table, $(p<0 \cdot 05)$. Cellularity increased subsequently, in both groups (Table). Alkaline phosphatase expression was detected in 258 of 696 CA colonies $(37 \%)$ but was undetected in CS colonies ( 0 of 192) after 96 hours in primary culture. PAS stains were negative in all CA and CS colonies.

\section{IN VIVO GRAFTING STUDIES}

In temporal morphogenesis studies, CA grafts which were retrieved at staggered intervals showed a well defined sequence of morphological changes during the formation of small intestinal neomucosa. At three days after transplantation, CA grafts had formed simple tubular structures lined by two cell layers of undifferentiated epithelium (Fig 4A). By five days, primitive cysts with foci of disorganised hyperchromatic cells at one pole had emerged (Fig 4B). By seven days, cysts had enlarged and, in some, hyperchromatic epithelium had formed a polarised single layer, confined to one edge. Other cysts showed early crypt formation (Fig 1C). By 10 days, villus structures and abundant goblet cells were present, in

Figure 4: Temporal neomucosal morphogenesis in subcutaneous grafts of intestinal cell aggregates $(C A)$. (A) $C A$ grafts three days after transplantation comprise tubular structures lined by one or two layers of undifferentiated hyperchromatic epithelium (arrowed). Grafted epithelium is surrounded by connective and some vascular tissue (haematoxylin and eosin stain, magnification $\times 175$; bar, $20 \mu \mathrm{m})$. (B) $C A$ grafts five days after transplantation. The tubular structures have developed a more prominent central lumen and stratified hyperchromatic cells have become polarised to one edge (arrowed) (haematoxylin and eosin stain, magnification $\times 450$; bar, $40 \mu \mathrm{m}$ ). (C) CA grafts seven days after transplantation. Enlargement of the central lumen has occurred to produce cyst-like structures. In simpler cysts, hyperchromatic epithelium has become localised to one edge (arrowed). In more developed cyst-like structures, crypt formation $(C)$ has occurred and seems to arise predominantly at one pole. Some goblet cells are present and mucin is seen within cyst-like spaces (haematoxylin and eosin stain, magnification $\times 75$; bar, $1200 \mu \mathrm{m})$. (D) $C A$ grafts at 10 days after transplantation. Many crypts (C) and rudimentary villi $(V)$ are evident. Many goblet cells are clearly seen (arrowed) (haematoxylin and eosin stain, magnification $\times 150 ;$ bar, $20 \mu \mathrm{m}$ ). 

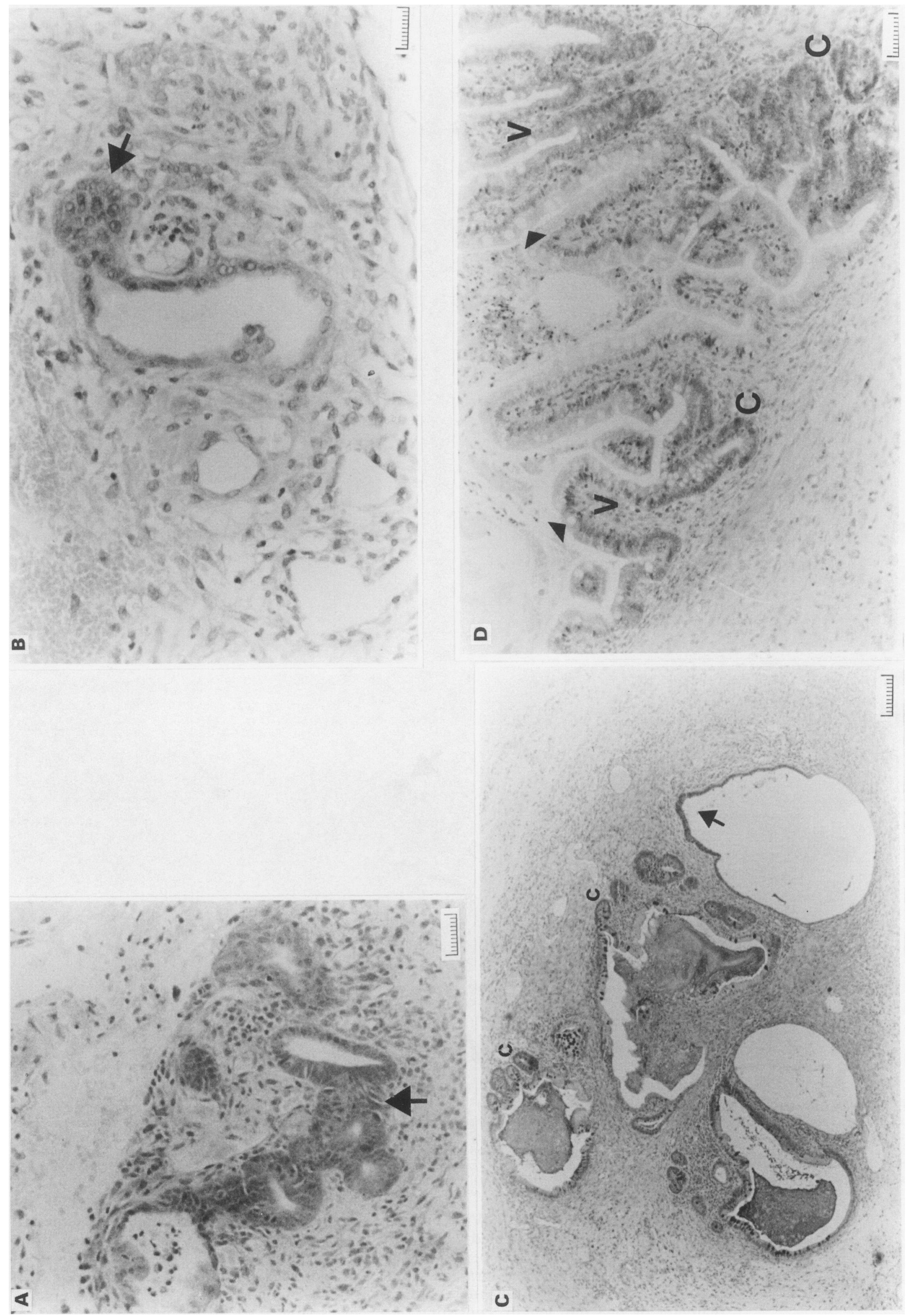

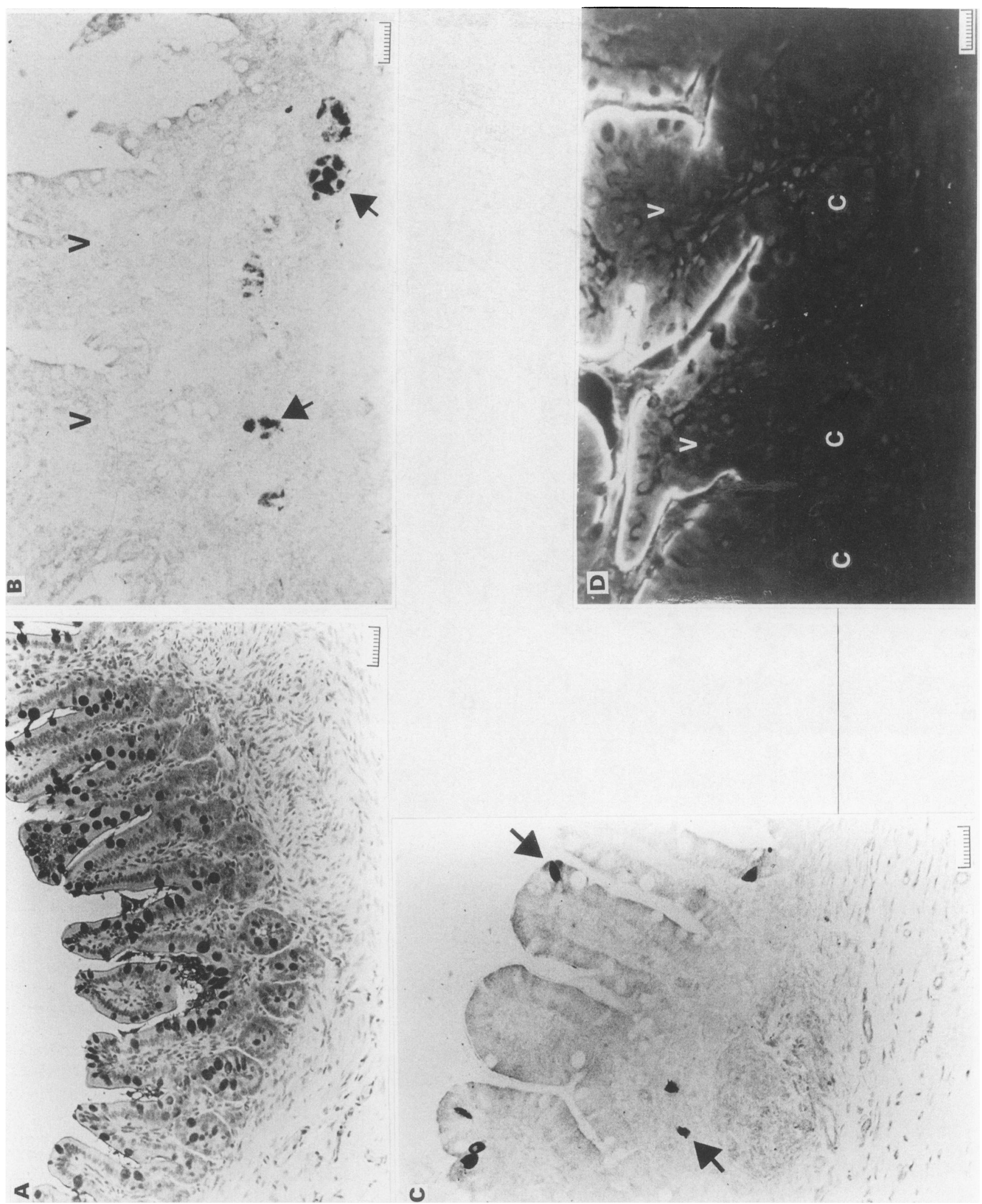

Figure 5: Cytodifferentiation in neomucosa induced by cell aggregate $(C A)$ grafts 14 days after transplantation. $(A)$ Goblet cells are identified by periodic acid Schifflalcian blue (showing dark) in both crypts and villi (magnification $\times 150 ;$ bar, $20 \mu \mathrm{m})$. (B) Paneth cells identified by polyclonal anti-lysozyme antibody. Note staining only occurring in the crypt region and not in the villi $(V)$-arrowed (magnification $\times 275 ;$ bar, $35 \mu m$ ). (C) Entero-endocrine cells identified by polyclonal anti-serotonin antibody arrowed (magnification $\times 250 ;$ bar, $35 \mu \mathrm{m}$ ). (D) Brush border alkaline phosphatase on absorptive enterocytes identified using Vector red substrate, and visualised through a rhodamine filter. Expression is confined to apical villus epithelium only (V), with no enzyme expression in the crypts (C) (magnification $\times 250$; bar, $35 \mu \mathrm{m}$ ).

addition to crypts (Fig 4D). With progression, cyst numbers declined but size increased, possible as a result of cyst coalescence. By 14 days, grafts had formed a single tubular structure, measuring to $2-3 \mathrm{~mm}$ diameter by $6-8 \mathrm{~mm}$ length, consisting of a single central lumen surrounded circumferentially by small intestine like neomucosa, bearing crypts, villi, and all epithelial cell lineages (goblet cells (Fig 5A), paneth cells (Fig 5B), entero-endocrine cells (Fig $5 \mathrm{C}$ ), and enterocytes (Fig 5D)). In the normal postnatal rat 


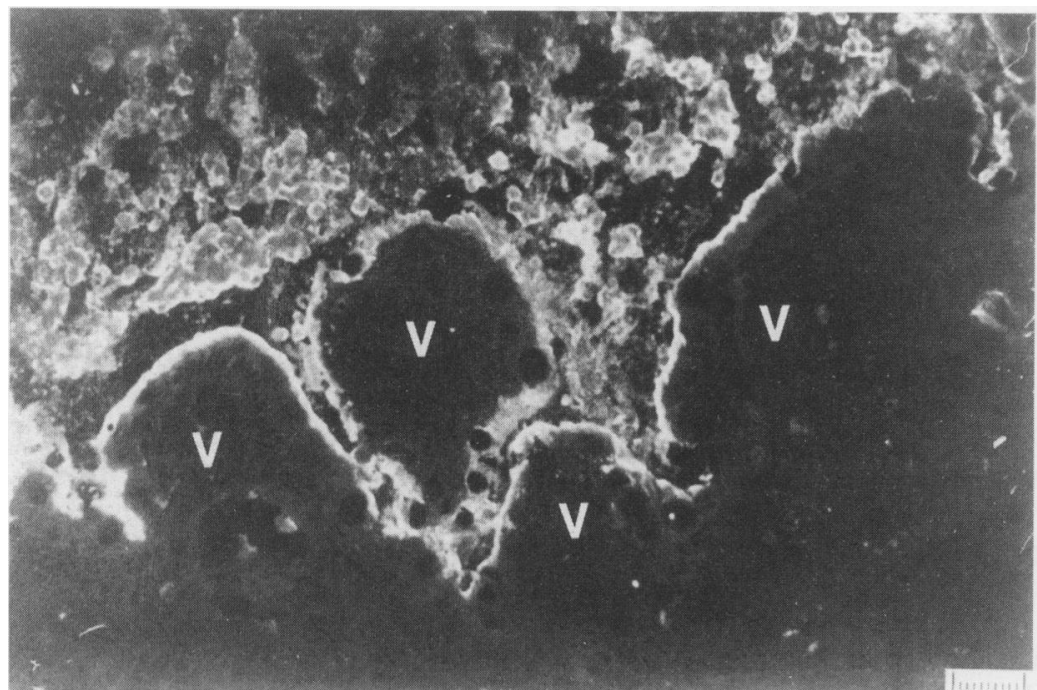

Figure 6: Neomucosa 14 days after grafting stained with fluorescent monoclonal antibody against sucrase. Sucrase expression (white) detected along the apical surface of neomucosal enterocytes ( $V-$ villus) (magnification $\times 225$; bar, $32 \mu \mathrm{m}$ ).

small intestine, sucrase expression depended on age. This enzyme was absent from 6 day old, normal small intestine but was detected between 14 and 21 days. Sucrase was also detected in neomucosa at 14 days after grafting (Fig 6).

In total, 48 of $56(87 \%)$ CA transplants produced successful neomucosal grafts. No palpable subcutaneous lump was found after CS grafting or sham procedures. Histological examination of CS or sham graft sites showed no evidence of subcutaneous epithelial cells, cellular growth, or intestine-like structures.

\section{Discussion}

The distinctive morphology of the small intestinal mucosa ensures a maximal surface area for absorption and enables interactions between the heterologous tissues elements which are necessary for optimal function. Inflammatory, infective, or chemical injury to the intestinal mucosa may disrupt these morphological arrangements with loss of barrier function and subsequent septic or immunological sequelae. However, the integrity of the intestine may be rapidly restored in adverse circumstances by mucosal regeneration. Indeed, mucosal regeneration is a principal feature of common inflammatory or ulcerative intestinal conditions.

Study of the relevant cell kinetic and structural changes in mucosal regeneration has been enhanced by the development of model systems. In 1968, Withers and Elkind induced intestinal mucosal damage and regeneration in intact rodents by high dose radiation. ${ }^{5}$ This method was modified by Potten and Henry in 1985 to involve lower dose radiation and simpler operational procedures. ${ }^{3}$ Most intestinal crypt stem cells were killed by irradiation and functional enterocytes subsequently expired over several days, leading to extensive epithelial necrosis and ulceration. Crypt numbers declined from approximately 120 to between 1 and 2 per small bowel circumference within three days of irradiation. ${ }^{3}$ Surviving progenitor cells within crypts mounted a proliferative response. Microcolonies, which were defined as groups of 10 or more healthy looking chromophilic cells, were formed. Each of these were considered to arise from an individual surviving crypt progenitor unit and they could be counted to give an index of crypt radiosensitivity. Microcolonies acquired a simple lumen, developed into crypt like structures, then multiplied by a process of crypt fission. Microcolonies enlarged to form macrocolonies which became visible to the naked eye. These subsequently coalesced with restoration of the epithelial layer and development of a complete crypt/villus architecture. Neomucosa, thus induced expressed all differentiated epithelial cell lineages and persisted throughout the life of the animal. ${ }^{11}$ Similar responses were observed after administration of cytotoxic agents. ${ }^{1}$ These models have shown component processes of the regeneration response - that it, colony formation, epithelial proliferation, three dimensional remodelling during neomucosal morphogenesis, and cytodifferentiation. However, the methods were limited by toxicity and early animal mortalities ${ }^{3}$ impeded prolonged study.

Evans et al have shown previously that crypt CA may be isolated from postnatal small intestinal mucosa by gentle enzymic digestion, then cultured in vitro. ${ }^{9} \mathrm{CA}$ isolated by this method may be grafted to induce neomucosa, in vivo. ${ }^{7812}$ The present study has shown that these methods also provide a novel model of mucosal regeneration with in vitro and in vivo components. Primary culture of CA enables assessment of colony formation and growth, by disaggregated intestinal epithelium. This technique may be useful for stringent assessment of regulatory factors, under controlled in vitro conditions. Cytodifferentiation in vitro, however, was very limited and only $37 \%$ CA colonies expressed alkaline phosphatase after 96 hours. Expression of this enzyme seemed to be unrelated to CA colony size and further work is necessary to elucidate relevant regulatory factors.

The in vivo arm of the model involved subcutaneous grafting of CA and showed a well defined temporal sequence of neomucosal morphogenesis and cytodifferentiation. Hence, processes may be studied in conditions uncomplicated by toxicity, stress responses, necrotic cell debris, and early animal mortality which are inherent to applied intestinal injury techniques. Furthermore, the epithelial grafts in our model may be more easily retrieved in isolated form at different stages of regeneration for detailed study.

The phenomenon of regeneration shown in our model bears some similarities to processes of fetal intestinal ontogenesis. Early fetal rat endoderm is the precursor of the intestinal epithelium, and at 14 days after conception it comprises stratified undifferentiated cells. High proliferative activity and active remodelling occurs subsequently. Intercellular or intracellular vacuoles develop and form into secondary lumina. The endoderm becomes converted from a stratified to a single polarised layer of cells. Lumina or vacuoles coalesce, 
leaving villus outgrowths. ${ }^{13}$ Crypts develop from epithelial downgrowth into the mesenchyme layer. ${ }^{14}$ Postnatal neomucosal morphogenesis, as shown in our model, is characterised in the early stages by conversion from double layered or disorganised epithelium to a single stratum of polarised cells, by coalescence of cyst or vacuole like structures to give a single central lumen, and by epithelial downgrowths with formation of crypts. Hence, these phenomonological similarities raise the possibility that the processes could share common regulatory factors.

Extensive experimental work has shown that cell-cell interactions, particularly between epithelium and mesenchyme, enhance the processes of growth, cytodifferentiation, morphogenesis, and functional specialisation during fetal intestinal development. ${ }^{15-19}$ Isolated fetal endoderm may actively recruit mesenchyme interactions from recipient tissues when grafted into a host animal. ${ }^{20}$ In contrast to this abundance of evidence, there are few data to the influence of cell interactions on postnatal mucosal regeneration.

We have previously shown that CA isolated by the method described contain epithelial and mesenchymal elements in close approximation. These aggregates of heterotypic cells have high regenerative potential. ${ }^{7812}$ In the present study, the relevance of this morphological cell-cell contact to mucosal regeneration was investigated. CA were subjected to a second digestion step, using trypsin and EDTA. This effectively disrupted any physical cell interactions and produced single CS, confirmed by light microscopy. Cell viability within CA and CS as indicated by the MTT assay, were similar.

In our model, however, CA had clear advantages. CA formed more numerous colonies with greater cellularity in vitro. Indeed, CA colonies had large multicellular epicentres which could represent the remnants of an individual cell aggregate that attached in culture. In the in vivo arm, CA grafts induced neomucosa with a small intestinal phenotype bearing all appropriate cell lineages. The enzyme sucrase, which is absent from normal intestine at birth and appears within 14-21 days, was detected in graft tissue at 14 days after transplantation. This suggests appropriate postnatal maturation of graft tissue. In contrast, CS formed sparse colonies which had smaller cellular epicentres. These could have arisen from reaggregation of separate cells in vitro or alternatively could represent attachment of smaller incompletely disaggregated cell clumps which were undetected on light microscopy. Once formed, CS colonies proliferated but none expressed alkaline phosphatase within 96 hours. All CS grafts failed in vivo. These results suggest that cell interactions within $C A$ may be relevant to in vitro colony formation, alkaline phosphatase expression, and in vivo neomucosal morphogenesis by disaggregated intestinal epithelium. These findings support the view that these cell interactions may influence intestinal tissue beyond fetal life. No attempt was made to seek individual effects of epithelial/epithelial or epithelial/mesenchymal cell interactions or specific molecular mechanisms of the regeneration response in this study. However, we intend to address these issues in subsequent studies.

In summary, the model described in this study enables assessment of elementary processes of postnatal mucosal regeneration. Within the model, preservation of cell-cell contact within cell aggregates enhances in vitro colony formation, expression of alkaline phosphatase, and temporal in vivo mucosal morphogenesis.

These studies were funded by the Wellcome Trust, Tayside Health Board, and the Medical Research Council. HRHP receives a Medical Research Council, Clinical Training Fellowship (No G84/3286). This assistance is gratefully acknowledged.

1 Al-Dewachi HS, Wright NA, Appleton DR, Watson AJ. The effect of a single injection of hydroxyurea on cell population kinetics in the small bowel mucosa of the rat Cell Tiss Kinet 1977; 10: 203-13.

2 Al-Dewachi HS, Wright NA, Appleton DR, Watson AJ The effects of a single injection of cytosine arabinoside on cell population kinetics in the mouse jejunal crypt. Virchows Arch (B) 1980; 34: 299-309.

3 Potten CS, Hendry JH. The microcolony assay in mouse small intestine. In: Potten CS, Hendry JH, eds. Cell clones: Manual of mammalian cell techniques. Edinburgh: Churchill-Livingstone, 1985: 50-60.

4 Withers HR, Elkind MM. Dose survival characteristics of epithelial cells of mouse intestinal mucosa. Radiology 1968; 91: 998-1000.

5 Withers HR, Elkind MM. Radiosensitivity and fractionation response of crypt cells of mouse jejunum. Radiat Res 1969; 38: 598-613.

6 Withers HR, Elkind MM. Microcolony survival assay for cells of mouse intestinal mucosa exposed to radiation Int f Radiation Biology 1970; 17: 261-8.

7 Tait IS, Penny JI, Campbell FC. Does neomucosa induced by small bowel stem cell transplantation have adequate function? Am f Surg 1995; 169: 120-5.

8 Tait IS, Flint N, Campbell FC, Evans GS. Generation of neomucosa in vivo by transplantation of dissociated postnatal small intestinal epithelium. Differentiation 1994; 56: natal small

9 Evans GS, Flint N, Somers AS, Eyden B, Potten CS. The development of a method for the preparation of rat intestinal epithelial cell primary cultures. $\mathcal{F}$ Cell Sci 1992; 101 219-31

10 Mosmann T. Rapid colorimetric assay for cellular growth and survival: Application to proliferation and cytotoxicity assays. F Immunol Methods 1983; 65: 55-63.

11 Cairnie AB, Millen BH. Fission of crypts of the small intestine of irradiated mouse. Cell and Tissue Kinet 1975; 8: 189-96.

12 Tait IS, Evans GS, Flint N, Campbell FC. Colonic mucosal replacement by syngeneic small intestinal stem cell transreplacement by syngeneic smal intestinal

13 Mathan M, Moxey PC, Trier JS. Morphogenesis of fetal rat duodenal villi. Am $\mathcal{F}$ Anat 1976; 146: 73-92.

14 Moxey PC, Trier JS. Specialised cell types in the human fetal small intestine. Anat Rec 1978; 191: 269-85.

15 Kedinger M, Simon PM, Grenier JF, Haffen K. Role of epithelial-mesenchymal interactions in the ontogenesis of intestinal brush border enzymes. Dev Biol 1981; 86: 339-47.

16 Kedinger M, Simon-Assman P, Lacroix B, Marxer A, Hauri HP, Haffen K. Fetal gut mesenchyme induces differentiation of cultured intestinal endodermal and crypt cells. Dev Biol 1986; 113: 474-83.

17 Bernfield M, Banerjee SD, Koda JE, Rapraeger AC. Remodelling of the basement membrane as a mechanism of morphogenetic tissue interaction. In: The role of extra-
cellular matrix in development. New York: A R Liss Inc,

18 Ekblom P. Developmentally regulated conversion of mesenchyme to epithelium. FASEB f 1989; 3: 2141-50

19 Colony PC. Successive phases of human fetal intestina development. In: Kretchmer N, Minkowski A, eds Nutritional adaptation of the gastrointestinal tract of the newborn. Vevey/New York: Nestlé/Raven Press, 1983. 3-28.

20 Del Buono R, Fleming KA, Morey AL, Hall PA, Wright NA. A nude mouse xenograft model of fetal intestine development and differentiation. Development 1992; 114: 67-73. 